\title{
Best of both worlds: promise of combining brain stimulation and brain connectome
}

\author{
Caroline Di Bernardi Luft' ${ }^{1}$, Ernesto Pereda ${ }^{2}$, Michael J. Banissy ${ }^{1}$ and Joydeep Bhattacharya ${ }^{1 *}$ \\ ' Department of Psychology, Goldsmiths, University of London, London, UK \\ ${ }^{2}$ Lab. of Electrical Engineering and Bioengineering, Department of Industrial Engineering, Institute of Biomedical Technology, University of La Laguna, Tenerife, \\ Spain
}

\section{Edited by:}

Mikhail Lebedev, Duke University, USA

Reviewed by:

Kohitij Kar, Rutgers University, USA

Diego Kaski, Imperial College

London, UK

*Correspondence:

Joydeep Bhattacharya, Department of Psychology, Goldsmiths,

University of London, New Cross,

London SE14 6NW, UK

e-mail: j.bhattacharya@gold.ac.uk
Transcranial current brain stimulation (tCS) is becoming increasingly popular as a non-pharmacological non-invasive neuromodulatory method that alters cortical excitability by applying weak electrical currents to the scalp via a pair of electrodes. Most applications of this technique have focused on enhancing motor and learning skills, as well as a therapeutic agent in neurological and psychiatric disorders. In these applications, similarly to lesion studies, tCS was used to provide a causal link between a function or behavior and a specific brain region (e.g., primary motor cortex). Nonetheless, complex cognitive functions are known to rely on functionally connected multitude of brain regions with dynamically changing patterns of information flow rather than on isolated areas, which are most commonly targeted in typical tCS experiments. In this review article, we argue in favor of combining tCS method with other neuroimaging techniques (e.g., fMRI, EEG) and by employing state-of-the-art connectivity data analysis techniques (e.g., graph theory) to obtain a deeper understanding of the underlying spatiotemporal dynamics of functional connectivity patterns and cognitive performance. Finally, we discuss the possibilities of using these combined techniques to investigate the neural correlates of human creativity and to enhance creativity.

Keywords: tCS, connectome, graph theory, functional connectivity, structural connectivity, tDCS, tACS, tRNS

\section{INTRODUCTION}

The possibility of non-invasively modulating the activity of the brain using transcranial current brain stimulation (tCS) has been intriguing the researchers in a variety of fields as it allows to improve cognition in various domains (Fregni et al., 2005; Santiesteban et al., 2012; Schaal et al., 2013; Snowball et al., 2013) or treat many human psychiatric conditions (Boggio et al., 2007, 2008; Rigonatti et al., 2008; Nitsche et al., 2009; Terhune and Cohen Kadosh, 2013). There are a number of tCS techniques available, including, but not limited to, transcranial direct current stimulation (tDCS), transcranial alternating current stimulation (tACS), and transcranial random noise stimulation (tRNS) (for a review on the tCS methods, see: Nitsche et al., 2008; Ruffini et al., 2013). In tDCS, a small direct current (DC) is passed from anodal (positive) to cathodal (negative) electrodes positioned in the head surface in order to target specific brain areas underneath the electrodes (Nitsche and Paulus, 2000; Faria et al., 2011). Early studies with animals demonstrated an increase in excitation through membrane depolarization in the neurons underneath anodal electrode but an inhibition under the cathodal one (Bindman et al., 1962, 1964; Purpura and McMurtry, 1965). In humans, there is evidence for an increase in excitability in areas underneath the anodal electrode and a decrease underneath the cathodal following tDCS on the motor (Nitsche and Paulus, 2000) and visual cortex (Antal et al., 2004). Although this rationale of higher excitability under anodal and inhibition under cathodal has been used for determining the stimulation protocol in many studies, it remains unclear if this is so in all cases, as other variables such as the position of the cathodal in relation to anodal (Nitsche and Paulus, 2000; Antal et al., 2004; Moliadze et al., 2010) and the intensity of the stimulation (Batsikadze et al., 2013) seem to interfere with the excitability effects observed under anodal and cathodal stimulation sites. In tRNS the areas underneath both electrodes are stimulated with a current whose amplitude varies randomly in time within the frequency range of $100-640 \mathrm{~Hz}$ (Terney et al., 2008; Ruffini et al., 2013). In tACS, an alternating current $(\mathrm{AC})$ with a pre-determined frequency passes from anodal to cathodal and the frequency is usually set within the EEG frequency spectrum $(1-100 \mathrm{~Hz}$ ) (Antal et al., 2008; Kanai et al., 2010).

The protocol for tCS stimulation, especially the anodal and cathodal electrodes location, is usually determined based on neuroimaging findings (e.g., EEG, fMRI) evidencing that a certain region is involved in the target brain function which the researcher wants to modulate. Therefore, most tCS studies hitherto are grounded on the modular paradigm, in which complex cognitive functions are thought to be mediated by independent brain areas (e.g., Kanwisher et al., 1997). Despite the great advance in the knowledge made through the modular paradigm in the last decades, the understanding that each cognitive function is mediated by independent brain areas is challenged by an increasing number of studies supporting that most cognitive 
functions are mediated by widely distributed areas functioning in parallel (Fuster, 2000; Sporns, 2014). For example, dyslexia was for a long time thought to be caused by a problem in the phonetic representations located in the primary and secondary auditory cortices (Goswami, 2000). However, recent work (Boets et al., 2013) has shown that dyslexic individuals have intact phonetic representations, but presented a problem in connectivity, both structural and functional, between inferior frontal gyrus (IFG) and the bilateral auditory cortex, which is associated with retrieving these representations. Other disorders such as schizophrenia (van den Heuvel et al., 2013), epilepsy (Bettus et al., 2008), and autism (Barttfeld et al., 2011) are also associated with abnormal (increased or decreased) brain connectivity rather than abnormal activity of isolated brain regions. In such cases, in seems logical that brain stimulation should not target one or the other isolated area, but the connection between them, which is certainly a challenging aim because most of the brain stimulation effects are assumed to be caused by the excitation/inhibition of the specific areas underneath anodal/cathodal electrodes.

Thus, in order to target specific connections rather than specific areas, it is necessary to understand how (or even whether) brain networks respond under or after tCS. In fact, the notion that tCS effects are brought about by increases/decreases in activation of the stimulated area has been challenged by studies showing that the effects of tDCS are not restricted to the stimulated sites (Lang et al., 2005; Kwon et al., 2008; Keeser et al., 2011). Moreover, there are some recent studies showing that tDCS affects brain connectivity patterns during both task and rest (Keeser et al., 2011; Polania et al., 2011a,b, 2012a; Meinzer et al., 2012, 2013), suggesting that the tCS has an impact not only on the target areas, but also on the brain networks. In this review paper, we discuss the possibility of tracking tCS-induced changes in the brain network by combining neuroimaging with advanced connectivity analysis techniques (e.g., graph theory). We briefly review the mechanisms of tCS and the basics of brain network analysis through graph theory as a framework to develop new brain stimulation protocols able to produce relevant changes in brain connectivity and, ultimately, in the features of a given brain network. In particular, we discuss the rationale for determining the stimulation protocol for improving creativity as an example of a complex cognitive construct which requires complex associations between multiple brain areas.

\section{POSSIBLE MECHANISMS OF tCS}

Before discussing the macro effects of tCS on connectivity, a brief discussion of its potential mechanisms is needed. Dissertating in detail on how the distinct tCS techniques can affect brain and behavior is out of the scope of this paper, but some of the key issues are elucidated here as they may be important for understanding how tCS can shape brain functional connectivity. Previous research with animals (Bindman et al., 1962, 1964; Purpura and McMurtry, 1965) showed that a small electrical DC passing through the anodal electrode can depolarize the cell membrane at subthreshold intensity, making the neurons more susceptible to excitatory activity as they become less negative, whereas the current passing the cathodal electrode polarizes the cell membrane making it more negative, inhibiting neural firing. Gartside (1968) found that a weak electrical current induced an increase in cortical firing under the stimulated area (anodal) in rats. Importantly, the same study observed that turning off the electrical current after $5 \mathrm{~min}$ of stimulation did not cease this increased neural discharge, termed the "aftereffect." Although in humans the currents reaching the brain through tCS are much weaker than the ones in animal studies (even if the external stimulating current is the same as humans have thicker skull), there is evidence that the brain areas underneath the anodal electrode are more prone to excitatory activity (Nitsche and Paulus, 2000, 2001; Antal et al., 2004). Repeated firing, or high-frequency stimulation, may result in long-term potentiation (LTP) and long-term depression (LTD) (Bliss and Lomo, 1973), which are thought to be the main mechanisms by which tCS modulates brain activity, as it does during learning (RioultPedotti et al., 2000). In the LTP process, sustained activation of the cell through the binding of glutamate to $\alpha$-Amino-3-hydroxy-5methyl-4-isoxazolepropionic acid (AMPA) receptors in the postsynaptic membrane causes the magnesium $\left(\mathrm{Mg}^{2+}\right)$ to leave the $\mathrm{N}$-methyl-D-aspartate receptor (NMDA) ion channel, allowing large quantities of calcium $\left(\mathrm{Ca}^{2+}\right)$ to enter the cell through this channel $\left(\mathrm{Mg}^{2+}\right.$ blocks the NMDA channel). These large quantities of $\mathrm{Ca}^{2+}$ in the post-synaptic dendrites can improve the synaptic efficiency for an extended time period by activating second messengers (calcium-dependent kinases such as the $\mathrm{Ca}^{2+} /$ calmodulin-dependent ones: CaMKs), which create more AMPA receptors and protein expressions (growth factor), thereby facilitating neural plasticity (Malenka and Bear, 2004). The role of LTP on tCS effects is supported by pharmacological studies showing that the administration of an antagonist of the NMDA receptor blocks the effects of anodal and cathodal stimulation on the motor evoked potential (MEP), as triggered by a transcranial magnetic stimulation (TMS) pulse, an indirect measure of motor cortex excitability (Liebetanz et al., 2002; Nitsche et al., 2003; Monte-Silva et al., 2013). Saturation of the LTP can induce LTD (Rioult-Pedotti et al., 2000), which might be one of the reasons why the tCS effects were found to be dosage dependant (Batsikadze et al., 2013). It is important to notice that the electrical currents delivered by tDCS/tACS and tRNS are not strong enough to fire an action potential (Radman et al., 2009), but they can cause a bimodal polarization effect, namely soma depolarization and apical dendrite hyperpolarization (Bikson et al., 2004). Therefore tCS, as opposed to TMS (Terao and Ugawa, 2002), affects the post-synaptic potential by promoting a change in the cell gain (Rahman et al., 2013), and not by increasing the firing itself. Furthermore, single neuron response to weak current stimulation seems to be rather dependant on the network dynamics. It was found that low frequency AC currents can induce changes in gamma oscillations $(25-35 \mathrm{~Hz})$ in a zero-sum fashion as increases in excitability were balanced by complimentary inhibitory activity according to the network dynamics (Reato et al., 2010). Other studies (Parra and Bikson, 2004; Deans et al., 2007) also revealed that the network plays a role even at the cellular level (in vitro studies), as the neuronal firing behavior was largely determined by the network it belongs to.

The ongoing neuronal oscillatory activity during stimulation seems to shape the effects of tDCS and tACS on the resulting 
network activity (Frohlich and McCormick, 2010; Ali et al., 2013; Frohlich and Schmidt, 2013). This appears to happen through a feedback loop between the neural activity and the endogenous electric field (Frohlich and McCormick, 2010). Thus, tACS at the endogenous oscillation frequency (in vitro) produced a higher enhancement of this oscillation as compared to tDCS (Ali et al., 2013). In addition, these authors found that the network oscillatory effects were more pronounced if the stimulated frequency matches the endogenous oscillation frequency, suggesting a resonance-like effect. The importance of network activity was not only evidenced at the cellular level, but also at the cortical level in awake human beings. In relation to tDCS, it was found that stimulating over the premotor cortex (PMC) resulted in increased excitability (as measured by the MEP) over the primary motor cortex (M1) (Boros et al., 2008), which suggests that stimulating one area can affect others structurally connected to it. This might explain why many studies found that the stimulation with weak currents affects a number of areas other than the region underneath the anodal electrode (Lang et al., 2005; Kwon et al., 2008; Keeser et al., 2011). The possibility that network dynamics also plays a role in the effects of tCS at the macro level is supported by studies showing that $\mathrm{tDCS}$ brings about changes in functional connectivity, especially when those changes are assessed during task performance (Polania et al., 2012a; Weber et al., 2014). The default mode network (DMN) (Keeser et al., 2011; Amadi et al., 2014) and the attention network (AN) (Pena-Gomez et al., 2012) also seem to be affected by brain stimulation, even when the stimulated area is not within the same network. However, studies investigating the effects of tCS on brain connectivity during different tasks are still scarce (Polania et al., 2011b, 2012a,b; Meinzer et al., 2012, 2013; Weber et al., 2014), but they may shed new light into how tCS affects the brain dynamics and behavior. One of the challenges on this enterprise is to develop a suitable framework that can guide not only the analysis and interpretation of complex connectivity results derived from various neuroimaging techniques, but also to develop more efficient protocols to tackle connectivity.

\section{NETWORK ANALYSIS AND BRAIN STIMULATION}

There are many ways of measuring how different brain areas interact or communicate with each other. Connectivity is usually investigated from three perspectives: structural, functional and effective (Friston, 2011). Structural connectivity refers to the anatomical connections within the brain, such as axons and synapses, that can be measured non-invasively through diffusion tensor imaging (DTI) (van den Heuvel and Sporns, 2013). The brain structure is thought to shape or determine the paths for the communication between brain areas while the brain is engaged in various tasks or even at rest (Friston, 2011). While structural connectivity traces the paths between regions based on the physical connections between them, functional and effective connectivity estimate these connections based on the relationship between the time series from each of these brain regions (voxels) or the corresponding electrode / sensor (van den Heuvel and Sporns, 2013). Functional connectivity refers to how interdependent the activity between two areas (or more) is, with no information on the direction of their communication, whereas effective connectivity refers to the directed (source and sink) connection between two areas (Horwitz, 2003; Friston, 2011). Both functional and effective connectivity are dynamic and can be measured from data collected using various neuroimaging techniques, such as EEG, MEG, and fMRI. A variety of algorithms has been developed to estimate functional (e.g., coherence, phase synchronization, correlation, synchronization likelihood), and effective (e.g., dynamic causal modeling, Granger causality, phase slope index) connectivity, and they are available in many neuroimaging analysis toolboxes (e.g., Delorme and Makeig, 2004; Oostenveld et al., 2011; Niso et al., 2013). These measures allow us to estimate the strength of the communication between regions/sensors and they all have different advantages and limitations (Pereda et al., 2005; Friston, 2011).

In order to understand the organizing principles of the brain networks (estimated using the techniques mentioned in the previous paragraph), we can use graph theory, which has emerged as an important model for understanding and quantifying the global properties of brain networks (Bassett and Bullmore, 2006; Bressler and Menon, 2010; Sporns, 2011). In graph theory, networks are mathematically represented as a set of nodes, which in this framework are the brain regions or the electrodes/sensors for EEG/MEG, connected through edges, which are the paths or lines representing the direct relation between the nodes (Figure 1A).

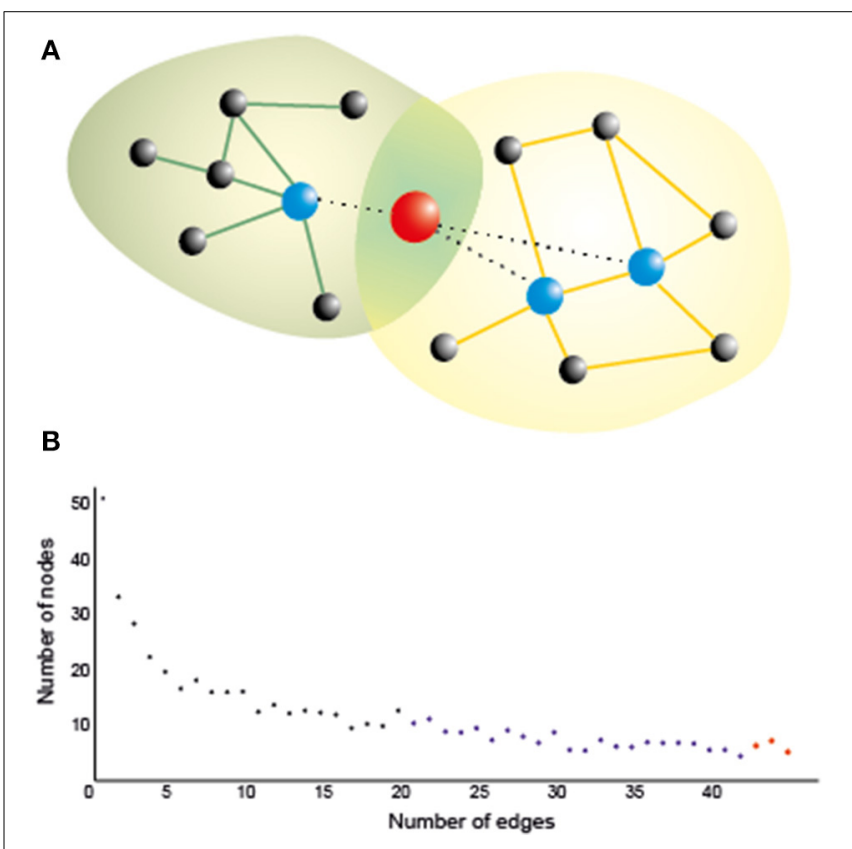

FIGURE 1 | (A) A hub naturally serves as a bridge between two or more networks, as it is connected to many nodes in all of them, thereby playing an important role in brain functioning. Stimulating a hub is likely to affect many nodes even at a long distance, which could maximize the effect of stimulation. Yet networks are also vulnerable against a directed attack on the hubs, so that their inhibition, however partial and/or transient, may crucially affect the corresponding cognitive functions if it is not properly controlled (e.g., by multisite stimulation of nearby nodes). Green and orange lines: intra-network edges. Dotted lines: edges connecting the hub with nodes in both networks: (B) A typical degree distribution for brain nodes as assessed by a functional connectivity index. Most of the nodes (black) have low degree (i.e., are connected only to a few nodes); some of them (blue) have moderate degree, and a few of them (in red) are hubs, which are connected to many nodes. 
Out of the many measures that can be used to characterize these networks (Rubinov and Sporns, 2010; Sporns, 2014), two of them are allegedly the most commonly used in neuroscience: (1) the average clustering coefficient, and (2) the average shortest path length. The clustering coefficient is the probability that neighboring nodes will be connected. The average shortest path length is the average minimum number of edges or connections that need to be traversed between two nodes. The arrangement of the edges in the network can take three main forms, which in turn determines the character of the network itself: regular, random, and small-world (Watts and Strogatz, 1998). In a regular network, each node is connected to its neighbors, resulting in high clustering and large average path length. If we randomly rewire most of the edges in a regular network, we would reduce the path length and the clustering coefficient, which characterizes a random network. However, if only a small number of edges are rewired to connect distant nodes, we have a "small-world" network, characterized by a small shortest path length and a high clustering coefficient (Watts and Strogatz, 1998). In small-world networks, the nodes containing the long-range connections have a similar number of connections in comparison to the other nodes, which makes the network resistant to random attack to the nodes. In the brain, however, there are some regions (termed network hubs) that are more heavily connected than others, and also more connected among themselves (van den Heuvel and Sporns, 2013). This type of network organization, with only a few nodes more connected than others and also more connected among themselves, is known as "scale-free," because such networks have a power law degree distribution (Barabasi and Albert, 1999; Sporns et al., 2004), which means that most nodes have only few connections or edges, whereas a few hubs have a large number of connections, as represented in Figure 1B. The presence of hubs (blue and red nodes in Figure 1), which are heavily connected and usually also centrally located nodes, and associated with locally connected specialized nodes, allows the network to have both local and global information processing (van den Heuvel and Sporns, 2011, 2013). The hubs are essential for brain communication, albeit energetically expensive as they are highly connected, and occupy a privileged position in the network, connecting distant communities of nodes to other hubs. In addition these hubs are organized as a "rich-club," in which densely connected nodes tend to be more connected to each other (van den Heuvel and Sporns, 2013). There are two types of rich-club hubs: connector hubs (red node Figure 1A), which interconnect different modules, and provincial hubs (blue nodes Figure 1A), which connect nodes within the same module. A study with DTI (van den Heuvel and Sporns, 2011) identified three main cortical areas (both hemispheres, near the midline) which are connector hubs: superior parietal, precuneus, and superior frontal cortex; and three subcortical regions (both hemispheres near medial regions) which are provincial hubs: putamen, hippocampus, and thalamus. Importantly, they found that attacking the rich-club connections (links between members of the rich-club) in comparison to random attacks or attacks to other hub connections caused a larger decrease in the global efficiency of the network. These hubs were defined based on structural connectivity, but there is accumulating evidence on the large overlap between brain network structure and functional connectivity especially during resting state (Cabral et al., 2014; Goni et al., 2014). Regions within the DMN, medially, contain most of the functional hubs, including most of the medial regions, including anterior cingulate cortex (ACC), precuneus/posterior cingulate gyrus. Using a measure of global functional connectivity, a study (Cole et al., 2010) found that not only the DMN regions have high functional connectivity with all other regions, but the cognitive control network (CCN) (Cole and Schneider, 2007), which comprises the dorsolateral prefrontal cortex (DLPFC), rostrolateral prefrontal cortex (RLPFC), dorsal-caudal ACC, inferior frontal junction (IFJ), posterior parietal cortex (PPC), PMC, and anterior insular cortex (AIC), has also high global connectivity. The regions in the DMN and in the CCN are among the top 5\% most connected regions in the brain (Cole et al., 2010). Recently, the concept of flexible hubs (Cole et al., 2013a,b), which are brain regions (e.g., DLPFC) that quickly shift their functional connectivity patterns (become highly connected) to implement cognitive control, has been discussed as an important part of network's ability for flexible behavior. Besides, a recent theoretical work (Aguirre et al., 2014) shows that communication through heavily connected nodes facilitates synchronization between different networks. Therefore, the structural and functional hubs seem to be an essential part of the scale-free brain network organization.

Making use of its structural and functional hubs, which can flexibly adapt to different environmental demands, the scalefree configuration allows dynamical exchange of information that facilitates parallel processing and rapid changes on its own configuration (Bassett and Bullmore, 2006). There is evidence that the scale-free network characteristics, as measured by graph theory, are optimized during awake compared to sleep periods (Uehara et al., 2014), suggesting that the functional organization of the network is relevant for cognitive processing. Therefore, graphtheory can be used to investigate structural, functional and effective connectivity. Functional connectivity gives undirected edges to the network, whereas effective connectivity provides information on the direction of the interactions investigated. Moreover, the edges can be weighted according to the degree of coupling between two nodes, as estimated by the techniques mentioned previously. Knowing and estimating the changes in network properties as a result of brain stimulation, during task and resting state alike, has important implications on the understanding of its effects over behavior. In addition, the knowledge of the networks representing the target cognitive process might provide insight into optimizing stimulation protocols.

\section{BRAIN STIMULATION AND CONNECTIVITY}

There is a great deal of research analysing the impact of TMS on brain connectivity patterns during task and rest (for a review, see Shafi et al., 2012). As TMS is not covered in this paper, we only summarize four main findings on TMS-induced alterations in connectivity, which can shed light onto how connectivity changes in response to brain stimulation: (1) state dependency: the brain state during stimulation affects how it modifies connectivity (Massimini et al., 2005; Davare et al., 2008; Morishima et al., 2009); (2) rich-club spreading: stimulating areas that have more connections (rich-club nodes) will affect a larger network 
(Bestmann et al., 2003, 2005; Chouinard et al., 2003); (3) structural connectivity spreading: stimulating one area affects other regions that are structurally connected to the main stimulated region (Pascual-Leone and Walsh, 2001; Mochizuki et al., 2004); (4) compensatory connectivity: inhibiting certain brain areas may trigger compensatory activity in the task related network (O'Shea et al., 2007). Although there are not many studies on the effects of tCS on brain connectivity, we will discuss some of these premises in the context of the few available ones published hitherto, which are listed in Table 1.

Most of the papers investigating the effect of tCS on brain connectivity analyzed the changes in the functional network during resting state (Alon et al., 2011; Keeser et al., 2011; Polania et al., 2011b, 2012a; Meinzer et al., 2012, 2013; Pena-Gomez et al., 2012). Studies vary in how they define the regions/nodes of the network. Using fMRI, some studies looked into the connectivity using M1 as a seed (Alon et al., 2011; Polania et al., 2012a; Sehm et al., 2013), while others looked into specific regions of interest (Pena-Gomez et al., 2012; Polania et al., 2012b; Chib et al., 2013; Weber et al., 2014), or into the resting state networks (Keeser et al., 2011; Pena-Gomez et al., 2012). Furthermore, graph-theory was also used for tracking connectivity changes after brain stimulation (Polania et al., 2011a).

The results on stimulating motor cortex (M1) are somehow mixed: some found increased functional connectivity within M1 (Polania et al., 2012a), whereas others found a decrease (Alon et al., 2011) or both a decrease during the stimulation but an increase afterwards (Sehm et al., 2013). One of the issues with these studies is that functional connectivity before and after stimulation was assessed during rest, yet M1 is not typically a region which is highly active during rest (Boros et al., 2008), so the impact of stimulation on brain connectivity may not be pronounced or strong as the motor network is relatively idle during rest. This possibility is supported by an EEG study on the effects of tDCS over M1 on functional connectivity during rest and during a finger tapping task (Polania et al., 2011a). These authors found that stimulation of the left motor cortex (anodal over C3/C5) with the cathodal electrode over right frontopolar electrodes (Fp2) was associated with higher connectivity of the motor areas in the gamma frequency band $(60-90 \mathrm{~Hz})$ during finger tapping. In addition, they found an increase in frontal connectivity in theta $(4-7 \mathrm{~Hz})$ and alpha $(8-12 \mathrm{~Hz})$ frequency bands during rest after tDCS stimulation, but this result was weaker than during task. They also found reduced coupling between frontal and occipital and areas after tDCS as compared to sham stimulation, which indicates that brain stimulation can shape connectivity not only by increasing communication between areas directly associated with the performing task, but also by reducing communication between other areas. Altogether, these results showed that the findings 1 (state-dependency) and 4 (compensatory connectivity) mentioned above can also explain some of the changes in functional connectivity following tDCS, while the aftereffects of the stimulation are still in place. The state-dependency in the cited studies is not related to the exact activity during the stimulation itself as referred in the TMS studies (Massimini et al., 2005; Davare et al., 2008; Morishima et al., 2009), but with the task conducted during the aftereffects of the stimulation. This means that the increase in coupling resulting from the stimulation is dependent on the task performed during the aftereffect period and on whether it recruits the stimulated network. In relation to finding 4 (compensatory connectivity), it seems that connectivity also changes in a zero-sum fashion, as it was suggested to be the case for most neuroenhancement interventions (Brem et al., 2014).

The state dependency seems to be important not only for tDCS, but also for tACS. By investigating how it can boost motion discrimination and lower adaptation, a study found that the method was only effective when the $10 \mathrm{~Hz}$ stimulation over the motion area (left hMT+) was administered during visual stimulation, but not before or after it (Kar and Krekelberg, 2014). This suggests that, differently from the tDCS, where the task conducted during the aftereffects can shape the effects of the stimulation, tACS effects on brain synchronization are dependent of the ongoing activity/task or brain state during which the stimulation is administered. In the latter study (Kar and Krekelberg, 2014), administering tACS during rest is unlikely to boost motion discrimination since the effects seem to be very dependent on the precise moment of visual perception in each trial.

The structural connections between brain areas, as in findings 2 (rich-club spreading) and 3 (structural connectivity spreading), also seem to be of importance for understanding how tCS affects the brain networks. It is possible to use the knowledge about the connectivity between areas to target deeper brain structures (Takano et al., 2011; Chib et al., 2013) which up until recently could only be targeted pharmacologically. Research with animals (Takano et al., 2011) observed an increased activation in the nucleus accumbens in rats after $10 \mathrm{~min}$ of stimulation over the frontal cortex. A recent study in humans (Chib et al., 2013) used tDCS to target midbrain areas (subcortical), including substantia nigra (SN) and the ventral tegmental area (VTA) by stimulating the ventral medial prefrontal cortex (VMPFC), during a face attractiveness judgment task, which is associated with the dopaminergic system for reward processing. The anodal and cathodal electrode locations were defined based on the knowledge that excitation of the VMPFC combined with inhibition of the DLPFC can bring about an increase in activity (together with dopamine release) of the midbrain (Takano et al., 2011). They found that the main stimulation groups (anodal over the VMPFC and cathodal over the DLPFC) increased their ratings of face attractiveness after the stimulation, but not the active sham group, which was stimulated for the same time, but with the opposite locations (anode over DLPFC and cathode over VMPFC). Importantly, the main stimulation elicited an increase in the BOLD signal in the midbrain areas and an increase in functional connectivity between VMPFC and the midbrain area. This increase in connectivity was correlated to the participants' ratings of attractiveness. In addition, the behavioral effect was only evident when the cathodal electrode was placed over the DLPFC, as the same effects were not found when the cathode was positioned on the vertex $(\mathrm{Cz})$. This result is consistent with the idea that coordinated activity between different brain areas, in this case VMPFC and DLPFC, can influence the outcomes of the stimulation in subcortical areas. Relevantly, it also demonstrates that it is possible to exploit the brain networks structure to target 


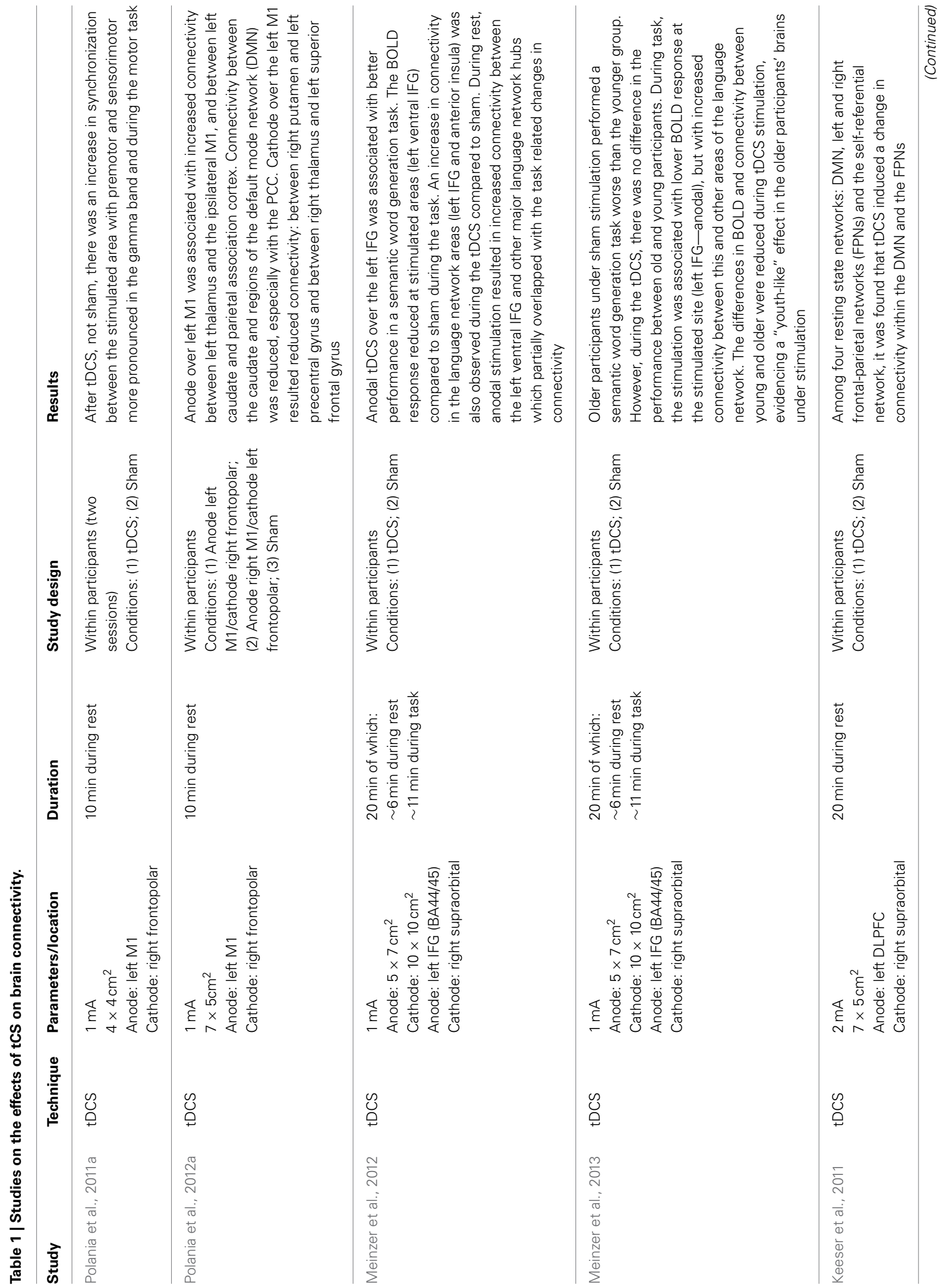




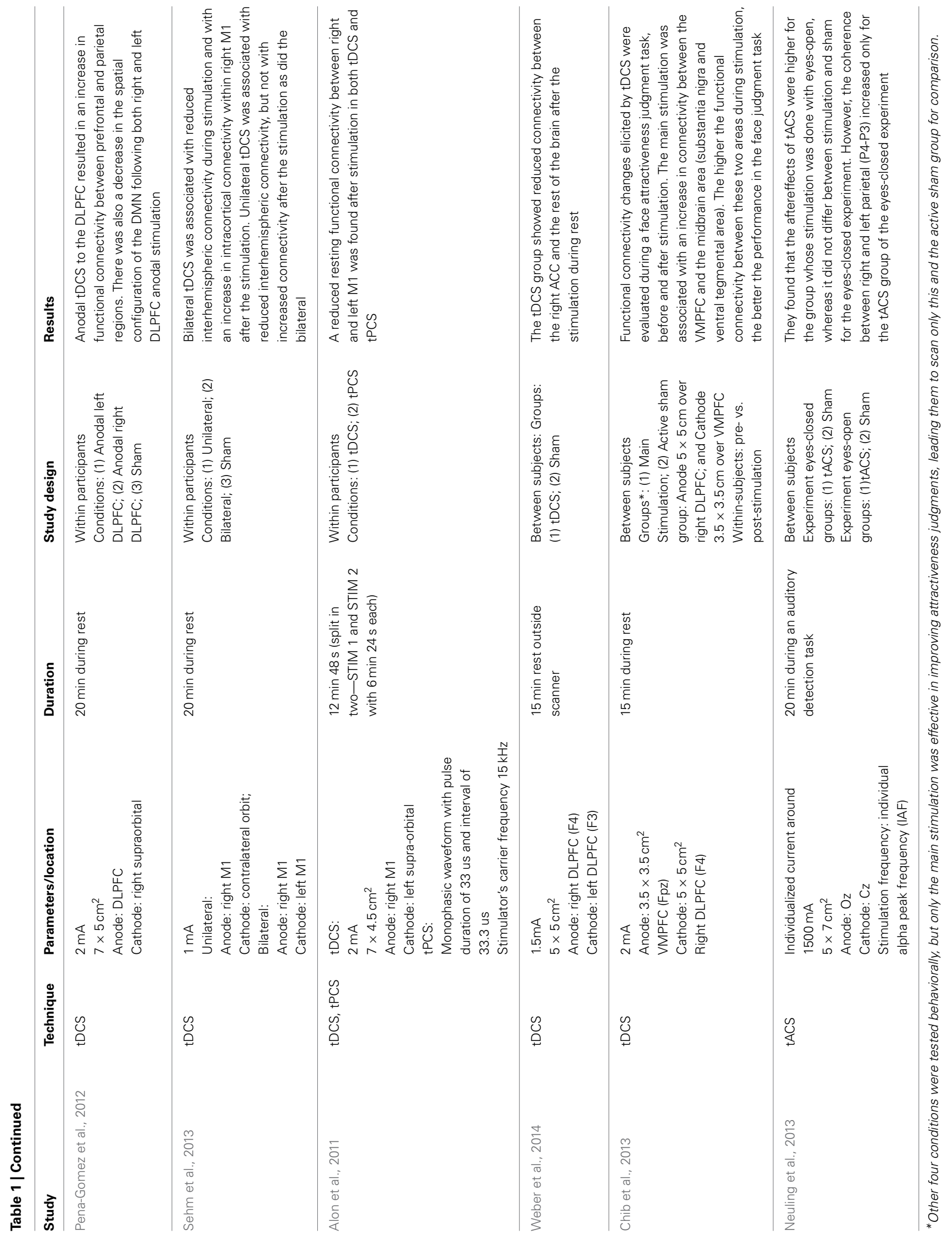


subcortical areas and their connections. The ability to affect connections between cortical and subcortical areas by stimulating the cerebral cortex has been also demonstrated during rest (Polania et al., 2012a).

Studies looking at resting state networks (Keeser et al., 2011; Pena-Gomez et al., 2012) found changes in functional connectivity after stimulation of the DLPFC. One of these studies (PenaGomez et al., 2012) found that stimulating either the right or the left DLPFC resulted in robust changes in the AN and in the DMN. It was observed that anodal tDCS over DLPFC was associated with a disruption in the DMN topography, as if the anterior (medial prefrontal) and posterior components (medial posterior) of the network become temporally independent after the stimulation. On the other hand, there was an increase in functional connectivity between frontal and parietal areas, which are part of the AN. An increase in fronto-parietal connectivity related to the AN was also found by others (Keeser et al., 2011), along with an increase in connectivity between an area near the anodal electrode on the left DLPFC and the DMN regions. It may be that tDCS over the DLPFC increases the alertness for action as indicated by the networks affected by stimulation, which it was suggested by the authors of both studies (Keeser et al., 2011; Pena-Gomez et al., 2012). As we mentioned in the previous section, the DLPFC can be considered a functional "flexible hub," which plays an important role in switching from one state to the other in order to attend to the necessary task demands (Cole et al., 2013b). This might be one of the reasons why stimulating DLPFC seems to affect functional connectivity in the DMN, as it reflects the change from one to the other network. These studies support that tCS can alter the connectivity in the brain during rest. In order to improve the interpretation of the results, new studies should target specific networks and bring new hypothesis of possible behavioral correlates. For example, what does it mean to increase or decrease connectivity in certain brain networks? Would these changes, for instance, improve mood, reduce depression, increase alertness, and others? The need for behavioral correlates in these studies is crucial for understanding their functional meaning.

Hitherto, two recent studies (Meinzer et al., 2012, 2013) used fMRI to monitor the brain activity during rest as well as during a semantic word generation task while the participants received anodal tDCS over the left IFG (targeting language areas-BA 44/45-see Table 1 for details). Both studies observed a reduction in the BOLD signal at the stimulated sites during the language task. Importantly, the connectivity between the stimulated area (left IFG) and other language-related area was increased during stimulation for both task and rest (Meinzer et al., 2012, 2013). It has been argued that these changes are related to increased neural efficiency at the stimulated site and its networks (Kar and Wright, 2014) similar to the changes observed as a result of learning (Buchel et al., 1999). These changes were found to be behaviorally relevant as the performance in the semantic word generation task improved when the participants were receiving active stimulation compared to sham. One of these studies (Meinzer et al., 2013) observed an interesting effect whereby older adults showed a pattern similar to that of their younger counterparts in terms of performance in the semantic task when receiving anodal stimulation over the left IFG, despite performing worse without stimulation. In a similar vein, the differences in BOLD activation and functional connectivity between young and older adults were reduced during anodal tDCS. In relation to the connectivity, older participants showed higher anterior (fronto-temporal, and medial frontal regions) and lower posterior (temporo-occipital, precentral, and postcentral cortices) functional connectivity than younger, which was related to worse task performance. Anodal tDCS stimulation over the left IFG seems to reverse these effects, as it reduced functional connectivity among anterior cortical areas and increased it among the posterior cortices (note that not all the age related connectivity differences were reversed though).

Considering that all but one of these studies (except Polania et al., 2011a), were conducted using tDCS in combination with fMRI, more research is needed using other tCS techniques, especially tACS and tRNS, combined with higher temporal resolution neuroimaging techniques such as EEG/MEG, as we cannot expect that all tCS techniques would impact brain connectivity in the same way. Nonetheless, there is an EEG study (Neuling et al., 2013 ) in which the participants' occipital cortex (anode: Oz) was stimulated in the individual alpha frequency (IAF) to compare the aftereffects of stimulating in two different conditions: eyesclosed and eyes-open. These authors found that alpha power only differs between tACS stimulation and sham in the eyesopen condition. The coherence between two parietal electrodes (P3-P4), however, was only increased after tACS stimulation with eyes-closed (not with eyes-open). Therefore, there was a difference in the aftereffects of stimulating the areas with eyes-open and eyes-closed, which support the idea that the effects of tCS are dependent on the brain state, and for tACS in particular, on the ongoing brain oscillations. Considering the time varying nature of the oscillatory brain activity, new real time protocols are being developed to adjust the stimulation frequency according to the brain oscillations in real time (Boyle and Frohlich, 2013).

\section{CREATIVITY, BRAIN STIMULATION, AND NETWORK ANALYSIS}

Creativity is a multidimensional construct that can be investigated from a number of different perspectives, from its associated processes such as convergent and divergent thinking (Sawyer, 2012), passing through the creative person, product and press or environment (Rhodes, 1961). In this paper, we borrowed the following definition of creativity from Plucker et al., 2004: "Creativity is the interaction among aptitude, process, and environment by which an individual or group produces a perceptible product that is both novel and useful as defined within a social context" ( $p$. 90). In the field of Cognitive Neuroscience, the main focus of creativity research is on the processes involved in the creative thinking, including divergent and convergent thinking (Luft and Bhattacharya, 2014). Divergent thinking refers to the capacity of generating novel and original ideas to open-ended problems (e.g., think of as many unusual uses of a brick). Convergent thinking, on the other hand, refers to the process of finding a correct solution to a closed-ended problem, such as a puzzle. In the real world, however, the creative process involves both divergent and convergent thinking (Sawyer, 2012). 
There are only a few studies on how we can boost creativity using tCS (Cerruti and Schlaug, 2009; Chi and Snyder, 2011, 2012; Metuki et al., 2012; Chrysikou et al., 2013), which are described in Table 2. On the divergent thinking study (Chrysikou et al., 2013), the authors positioned the cathodal electrode over the left or the right prefrontal cortex and the anode over the mastoid, in an attempt to inhibit the left or right DLPFC. They found that cathodal stimulation over the left DLPFC, but not over the right DLPFC, was associated with quicker responses on the uncommon uses task (the participants were asked to generate common or uncommon uses for presented objects). The authors suggested that this result is coherent with the idea that divergent thinking depends on transient hypo-frontality, but in this case, it was found to be specific to the left hemisphere.

Most of the studies cited in Table 2 investigated creative insight, which is a convergent thinking process. In one of the insight studies (Cerruti and Schlaug, 2009) it was found that anodal stimulation over the left DLPFC increases the solution rate in a convergent thinking task, the "Remote Associate Task-RAT." In this task, the participants have to find a fourth word which makes a compound word with three words presented on the screen (e.g., food/forward/break; solution: fast). However, another study (Metuki et al., 2012) observed that anodal stimulation over the left DLPFC did not improve the solution rate when subjects were given less time to solve the problem ( $7 \mathrm{~s}$ compared to $30 \mathrm{~s}$ ), but it did improve subjects' ability to recognize correct solutions to hard RAT problems. The authors suggested that the left DLPFC is involved in recognizing the correct solution rather than generating it, a role that has been attributed to the right hemisphere (Bowden and Jung-Beeman, 2003), especially the right anterior area (Jung-Beeman et al., 2004). A slightly different account can be suggested based on two experiments (Anderson et al., 2009) monitoring the subjects brain responses (fMRI) while they solved a compound-word RAT task (Experiment 1) and another similar paradigm which allowed faster responses (Experiment 2). They observed that while the LIPFC was associated with the word search in memory or memory retrieval, the ACC was associated with the processing of solutions. In both experiments, the LIPFC activity increased when the participants were trying to find a solution, but from the moment they reached it, the ACC activity increased and the LIPFC returned to baseline levels.

The other two insight studies (Chi and Snyder, 2011, 2012) focused on other insight problems that are less dependent on verbal processes (matchstick and 9-dot problems). In both studies (Chi and Snyder, 2011, 2012), the stimulation protocol positioned the cathodal electrode over the left anterior temporal lobe (ATL), and the anodal over the right ATL. The authors found that this protocol increased the solution rates to these problems in relation to sham stimulation (between groups).

Therefore, for insight studies, it seems that the left DLPFC, and the right ATL play a role in finding solutions for convergent thinking. Nonetheless, little is still known about how these areas communicate to generate a solution as none of these studies combined tCS with any neuroimaging techniques, making it difficult to know whether these changes in performance are indeed caused by the excited/inhibited areas (Chi and Snyder,
2011, 2012; Chrysikou et al., 2013), by a connection between the stimulated/inhibited area, or by a compensatory network mechanisms that may be triggered by inhibiting those (as in finding 4 described in the previous section). In addition, in the human motor system evidence that bilateral tDCS evokes a suppression of one hemisphere but a facilitation of the other is somewhat mixed (see Nitsche and Paulus, 2000; Mordillo-Mateos et al., 2012; Hasan et al., 2013). Thus, the biological impact of bilateral tDCS (and by virtue the mechanisms that modulate previously reported enhancements in creativity following bilateral tDCS-e.g., Chi and Snyder, 2011, 2012) remains unclear.

Previous studies on the brain activity underlying creativity found, for example, that divergent thinking is associated with higher functional connectivity between medial prefrontal cortex (mPFC) and posterior cingulate cortex (PCC), both of which are key nodes of the DMN (Takeuchi et al., 2012). It could be that inhibiting the lateral prefrontal cortex may result in higher activation of the medial prefrontal, but this can only be tested by combining tCS with neuroimaging. There are a number of studies suggesting that creativity is associated with higher connectivity, both structural (Jung et al., 2010a,b; Takeuchi et al., 2010) and functional during rest (Jaušovec and Jaušovec, 2000a; Kounios et al., 2008; Takeuchi et al., 2012) and task alike (Jaušovec and Jaušovec, 2000b; Bhattacharya and Petsche, 2002, 2005; Razumnikova and Larina, 2005). Further, there are recent suggestions that creative ideas may reside in dynamic activation patterns of spontaneous brain networks (Wiggins and Bhattacharya, 2014). On account of these works, we believe that an important step towards improving creativity through tCS would be to combine brain stimulation with neuroimaging and advanced analysis techniques, in order to find how the brain networks mediate the improvements in creative processes observed in the cited studies. Importantly, techniques such as tACS allow targeting both long range and local synchronization (Ali et al., 2013).

Based on the previous discussions, we propose a hypothetical approach for combining neuroimaging, connectivity and brain stimulation for improving creativity (Figure 2). In this approach, the research starts with the graph theoretical analysis of the connectivity between the regions involved in a certain process (e.g., convergent thinking-insight) as in Figure 2A. Using the discovered cognitive connections (Figure 2B), a protocol for stimulation is determined (Figure 2C). In Figure 2A, we showed a couple of regions involved in creativity. Immediately after the stimulation, the connectivity patterns (in identical conditions of the analyzed patterns in Figures 2A,B) are analyzed against control stimulation protocol, e.g., sham stimulation. The results are presented as a map, with the edges linking the nodes representing here the strength of the difference in connectivity between active and sham stimulation (or any other control or contrast of the experiment), as in Figure 2D. In Figure 2 hypothetical example, the connectivity changed after stimulation of the left DLPFC during a RAT paradigm, especially among the temporal and frontal areas (Figure 2D). In the hypothetical example, there was an increase in communication between ATL and DLPFC and between the DLPFC and the ACC, which could represent coordinated (DLPFC) search of the solution in memory (ATL) and 


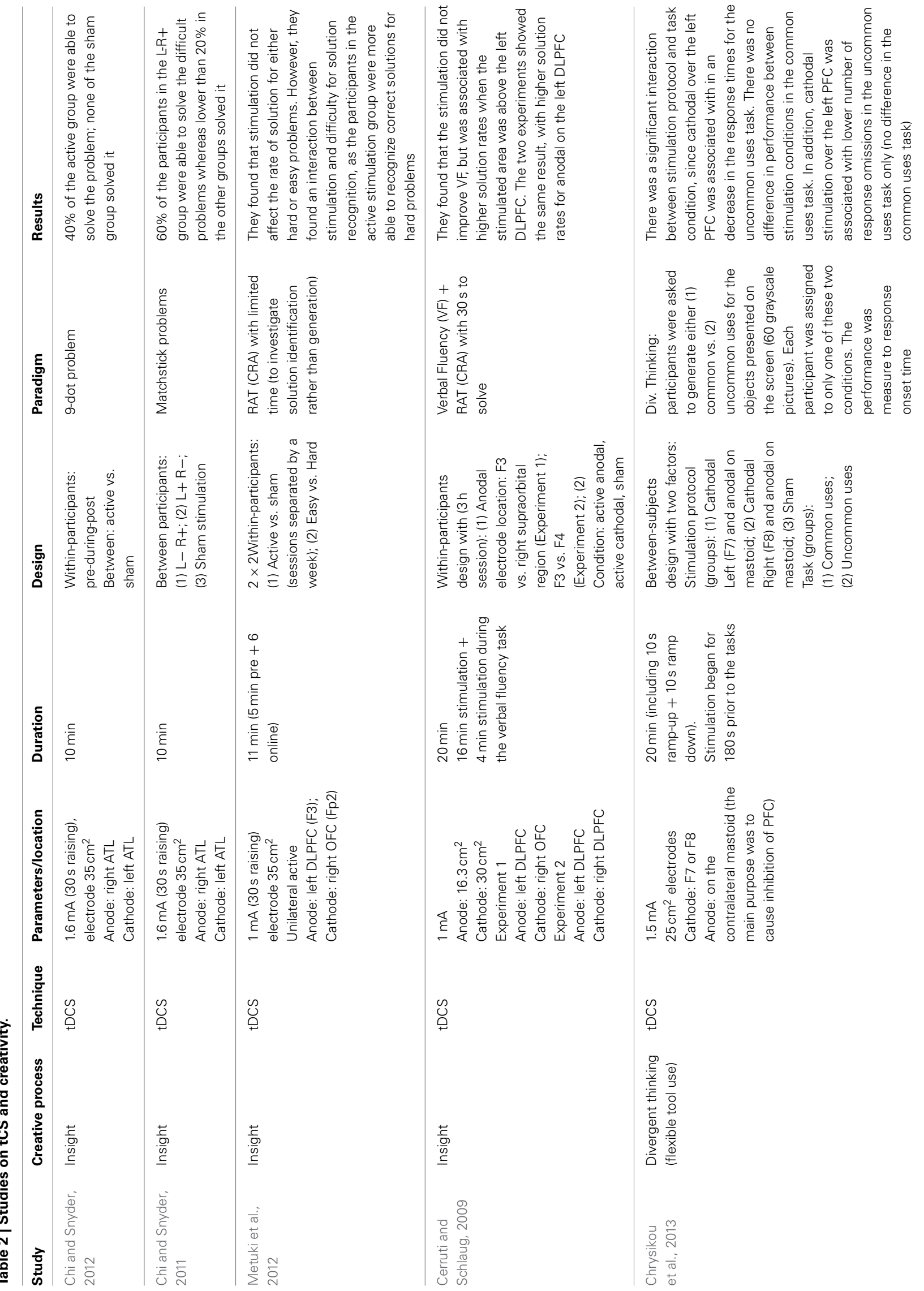


A

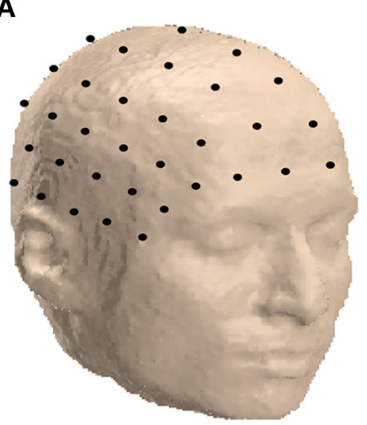

C

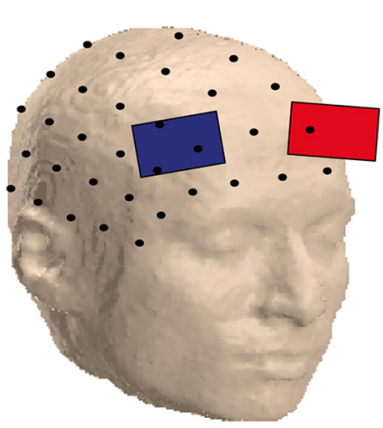

B

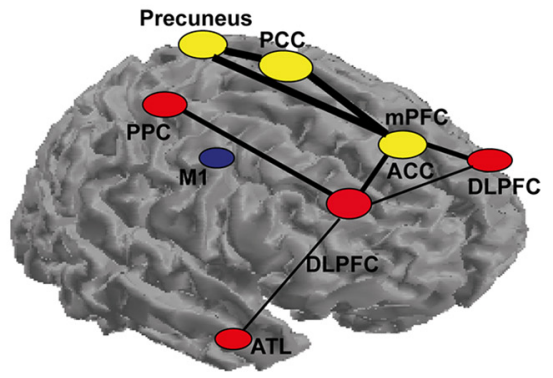

D

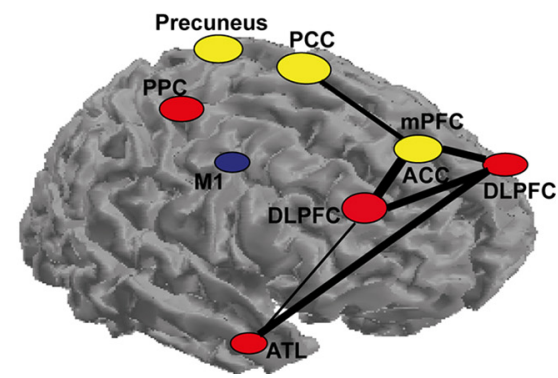

FIGURE 2 | Schematic representation of the possible combination of tCS and functional (EEG) brain connectivity to enhance creativity. An array of scalp electrodes, a subset of which is depicted in (A), is recorded. In (B) is possible to identify ROls which are highly connected (yellow) and the ones that are flexible hubs (red) or less connected areas (blue) during a solution vs. non-solution RAT task. This helps identifying the target electrodes to stimulate as in (C). Stimulating these electrodes may not only eliminate the differences for the corresponding nodes, but also reduce them for areas in the same network, which are not stimulated but functionally connected to the targeted ones as depicted in (D). It is worth noting that the labels and the networks drawn in the figure are only for demonstration, as they are not precisely equivalent to their anatomical locations (they are only approximate locations on a surface, the areas in yellow are located in the medial area of the brain, which cannot be seen in a cortical mesh). The areas are abbreviated as follows: PCC, posterior cingulate cortex; PPC, posterior parietal cortex; M1, primary motor cortex; mPFC, medial prefrontal cortex; ACC, anterior cingulate cortex; DLPFC, dorsolateral prefrontal cortex; ATL, anterior temporal lobe. the recognition of the correct solution (ACC), from DLPFC to ACC. Note that this approach does not account for the direction of the interactions, which could be also tested using effective connectivity analysis techniques such as dynamic causal modeling or Granger causality. In divergent thinking, the approach would probably result in a higher change over the posterior rather than anterior regions.

\section{LIMITATIONS OF GRAPH-THEORY AND BRAIN STIMULATION}

Notwithstanding the advances emerging from the combination of tCS and neuroimaging for the understanding of the connectivity changes in response to brain stimulation, there are many limitations of both methods which can be thought as new challenges for this enterprise. First, despite its usefulness in characterizing brain network during cognitive functions (Sporns, 2014), the application of graph theory is not problem-free (Fornito et al., 2013). The approach, after all, is based on sophisticated mathematical techniques that require judicious choices at various steps of the analysis. Perhaps the most obvious one is the need to choose among a number of possible strategies to reconstruct the networks, which do not always lead to a convergent or consistent outcome. For instance, one has to decide whether the links will be weighted or unweighted, directed or not, whether to use a fixed value or a fixed link density across participants as a threshold or to use statistical controls such as surrogate data analysis in order to determine the significance in a per-link basis. Further, especially in the case of EEG/MEG, many different bivariate indices of functional/effective connectivity are available (e.g., Niso et al., 2013). Besides, spurious characterization of a network could result from an inappropriate temporal and/or spatial sampling of the underlying systems (Bialonski et al., 2010, 2011). Last, but not least, the relationship between connectivity at the sensor/electrode level and connectivity at the neural source level is more complicated than traditionally assumed (Ewald et al., 2012). Nevertheless, we argue that graph theory is a very useful tool to study the synchronized activity between different brain areas underlying most cognitive functions, as it can be used to characterize these patterns of brain connectivity. Moreover, it can be also used to understand how local changes (whether internally or externally generated) are able to affect other distant brain areas. For instance, in focal epilepsy research a paradigm shift is currently taking place whereby attention is increasingly not toward the epileptic focus itself, the classical and obvious target area of the neuroscientific research on epilepsy, but the epileptic network and its characteristics (Lehnertz et al., 2014), offering a tremendous potential in minimizing the extent of surgical intervention.

The tCS methods also face limitations that have to be considered when attempting to develop protocols to boost specific cognitive functions. Currently, there are many issues related to our lack of understanding of how tCS, in its different modalities, 
shapes neural activity. First, as tCS methods in humans are non-invasive, the stimulation is applied to the skull rather than directly to the brain, meaning that the spatial resolution of tCS is diffuse due to skull dispersion. The spatial resolution can be improved with small stimulation electrodes (Datta et al., 2009), but it still does not overcome the problem that the stimulation is indirect and the electrodes often too large for such focal stimulation of a small cortical area. The second limitation, which is closely related to the first, is the lack of control of the stimulation current reaching the brain as the current is altered as it passes through the skull to be then conducted by the cerebrospinal fluid to the brain. Individual differences in skull thickness and shape may interact with how much current is actually reaching the brain (Datta et al., 2009). Therefore, even with advanced modeling of the current (Wagner et al., 2007; Shahid et al., 2014), it is difficult to predict how much current is actually reaching the brain. Third, the stimulation effects, as stated earlier in the Introduction, are not straightforward. For example, anodal stimulation does not always cause increase excitability, and vice versa for cathodal (Nitsche and Paulus, 2000; Antal et al., 2004; Moliadze et al., 2010). Further, other factors such as increasing the intensity (Batsikadze et al., 2013) or including a task during stimulation (Antal et al., 2007) can also change the effects in a complicated fashion. Therefore, it is important for these factors to be controlled carefully in tCS studies. Moreover, when one considers these limitations in conjunction with the fact that stimulating one area of the brain can affect a network of regions, it hampers the possibility of claiming "cause-effects" relations between a single stimulated brain region and specific cognitive functions as the stimulation effects are not entirely known.

\section{CONCLUSION}

In this paper, we discussed the possibility of combining tCS with neuroimaging and graph theory for analysing the impact of brain stimulation on brain connectivity. In doing so, we highlight how graph theoretical analysis can help in understanding how the brain networks are affected by tCS in specific locations. In addition, we suggest that the knowledge of structural connectivity pathways can be used to target a network of brain areas rather than a single area and that ongoing brain connectivity during and just after (during the aftereffects period) tCS is an important factor in determining the connectivity changes in response to stimulation. In the future, we suggest using graph theory not only to understand the network effects of stimulating different brain areas, but also to develop tCS protocols that can target connectivity rather than individual brain areas.

\section{ACKNOWLEDGMENTS}

The authors are supported by the CREAM project that has been funded by the European Commission under Grant Agreement no 612022. This publication reflects the views only of the authors, and the European Commission cannot be held responsible for any use which may be made of the information contained therein. Michael J. Banissy is also supported by the Economic and Social Research Council (ES/K00882X/1).

\section{REFERENCES}

Aguirre, J., Sevilla-Escoboza, R., Gutiérrez, R., Papo, D., and Buldú, J. M. (2014). Synchronization of interconnected networks: the role of connector nodes. Phys. Rev. Lett. 112:248701. doi: 10.1103/PhysRevLett.112.248701

Ali, M. M., Sellers, K. K., and Frohlich, F. (2013). Transcranial alternating current stimulation modulates large-scale cortical network activity by network resonance. J. Neurosci. 33, 11262-11275. doi: 10.1523/JNEUROSCI.5867-12.2013

Alon, G., Roys, S. R., Gullapalli, R. P., and Greenspan, J. D. (2011). Non-invasive electrical stimulation of the brain (ESB) modifies the resting-state network connectivity of the primary motor cortex: a proof of concept fMRI study. Brain Res. 1403, 37-44. doi: 10.1016/j.brainres.2011.06.013

Amadi, U., Ilie, A., Johansen-Berg, H., and Stagg, C. J. (2014). Polarity-specific effects of motor transcranial direct current stimulation on fMRI resting state networks. Neuroimage 88, 155-161. doi: 10.1016/j.neuroimage.2013.11.037

Anderson, J. R., Anderson, J. F., Ferris, J. L., Fincham, J. M., and Jung, K. J. (2009). Lateral inferior prefrontal cortex and anterior cingulate cortex are engaged at different stages in the solution of insight problems. Proc. Natl. Acad. Sci. U.S.A. 106, 10799-10804. doi: 10.1073/pnas.0903953106

Antal, A., Boros, K., Poreisz, C., Chaieb, L., Terney, D., and Paulus, W. (2008) Comparatively weak after-effects of transcranial alternating current stimulation (tACS) on cortical excitability in humans. Brain Stimul. 1, 97-105. doi: 10.1016/j.brs.2007.10.001

Antal, A., Kincses, T. Z., Nitsche, M. A., Bartfai, O., and Paulus, W. (2004). Excitability changes induced in the human primary visual cortex by transcranial direct current stimulation: direct electrophysiological evidence. Invest. Ophthalmol. Vis. Sci. 45, 702-707. doi: 10.1167/iovs.03-0688

Antal, A., Terney, D., Poreisz, C., and Paulus, W. (2007). Towards unravelling task-related modulations of neuroplastic changes induced in the human motor cortex. Eur. J. Neurosci. 26, 2687-2691. doi: 10.1111/j.1460-9568.2007.05896.x

Barabasi, A. L., and Albert, R. (1999). Emergence of scaling in random networks. Science 286, 509-512. doi: 10.1126/science.286.5439.509

Barttfeld, P., Wicker, B., Cukier, S., Navarta, S., Lew, S., and Sigman, M. (2011). A big-world network in ASD: dynamical connectivity analysis reflects a deficit in long-range connections and an excess of short-range connections. Neuropsychologia 49, 254-263. doi: 10.1016/j.neuropsychologia.2010.11.024

Bassett, D. S., and Bullmore, E. (2006). Small-world brain networks. Neuroscientist 12, 512-523. doi: 10.1177/1073858406293182

Batsikadze, G., Moliadze, V., Paulus, W., Kuo, M. F., and Nitsche, M. A. (2013). Partially non-linear stimulation intensity-dependent effects of direct current stimulation on motor cortex excitability in humans. J. Physiol. 591, 1987-2000. doi: 10.1113/jphysiol.2012.249730

Bestmann, S., Baudewig, J., Siebner, H. R., Rothwell, J. C., and Frahm, J. (2003). Subthreshold high-frequency TMS of human primary motor cortex modulates interconnected frontal motor areas as detected by interleaved fMRI-TMS. Neuroimage 20, 1685-1696. doi: 10.1016/j.neuroimage. 2003. 07.028

Bestmann, S., Baudewig, J., Siebner, H. R., Rothwell, J. C., and Frahm, J. (2005). BOLD MRI responses to repetitive TMS over human dorsal premotor cortex. Neuroimage 28, 22-29. doi: 10.1016/j.neuroimage.2005.05.027

Bettus, G., Wendling, F., Guye, M., Valton, L., Regis, J., Chauvel, P., et al. (2008). Enhanced EEG functional connectivity in mesial temporal lobe epilepsy. Epilepsy Res. 81, 58-68. doi: 10.1016/j.eplepsyres.2008. 04.020

Bhattacharya, J., and Petsche, H. (2002). Shadows of artistry: cortical synchrony during perception and imagery of visual art. Brain Res. Cogn. Brain Res. 13, 179-186. doi: 10.1016/S0926-6410(01)00110-0

Bhattacharya, J., and Petsche, H. (2005). Drawing on mind's canvas: differences in cortical integration patterns between artists and non-artists. Hum. Brain Mapp. 26, 1-14. doi: 10.1002/hbm.20104

Bialonski, S., Horstmann, M. T., and Lehnertz, K. (2010). From brain to earth and climate systems: small-world interaction networks or not? Chaos 20, 013134. doi: $10.1063 / 1.3360561$

Bialonski, S., Wendler, M., and Lehnertz, K. (2011). Unraveling spurious properties of interaction networks with tailored random networks. PLOS ONE 6:e22826. doi: 10.1371/journal.pone.0022826

Bikson, M., Inoue, M., Akiyama, H., Deans, J. K., Fox, J. E., Miyakawa, H., et al. (2004). Effects of uniform extracellular DC electric fields on excitability in rat hippocampal slices in vitro. J. Physiol. 557, 175-190. doi: 10.1113/jphysiol.2003.055772 
Bindman, L. J., Lippold, O. C., and Redfearn, J. W. (1962). Long-lasting changes in the level of the electrical activity of the cerebral cortex produced bypolarizing currents. Nature 196, 584-585. doi: 10.1038/196584a0

Bindman, L. J., Lippold, O. C., and Redfearn, J. W. (1964). The action of brief polarizing currents on the cerebral cortex of the rat (1) during current flow and (2) in the production of long-lasting after-effects. J. Physiol. 172, 369-382.

Bliss, T. V., and Lomo, T. (1973). Long-lasting potentiation of synaptic transmission in the dentate area of the anaesthetized rabbit following stimulation of the perforant path. J. Physiol. 232, 331-356.

Boets, B., Op De Beeck, H. P., Vandermosten, M., Scott, S. K., Gillebert, C. R., Mantini, D., et al. (2013). Intact but less accessible phonetic representations in adults with dyslexia. Science 342, 1251-1254. doi: 10.1126/science. 1244333

Boggio, P. S., Nunes, A., Rigonatti, S. P., Nitsche, M. A., Pascual-Leone, A., and Fregni, F. (2007). Repeated sessions of noninvasive brain DC stimulation is associated with motor function improvement in stroke patients. Restor. Neurol. Neurosci. 25, 123-129.

Boggio, P. S., Rigonatti, S. P., Ribeiro, R. B., Myczkowski, M. L., Nitsche, M. A., Pascual-Leone, A., et al. (2008). A randomized, double-blind clinical trial on the efficacy of cortical direct current stimulation for the treatment of major depression. Int. J. Neuropsychopharmacol. 11, 249-254. doi: 10.1017/S1461145707007833

Boros, K., Poreisz, C., Munchau, A., Paulus, W., and Nitsche, M. A. (2008). Premotor transcranial direct current stimulation (tDCS) affects primary motor excitability in humans. Eur. J. Neurosci. 27, 1292-1300. doi: 10.1111/j.14609568.2008.06090.x

Bowden, E. M., and Jung-Beeman, M. (2003). Aha! Insight experience correlates with solution activation in the right hemisphere. Psychon. Bull. Rev. 10, 730-737. doi: 10.3758/BF03196539

Boyle, M. R., and Frohlich, F. (2013). "EEG feedback-controlled transcranial alternating current stimulation," in 2013 6th International IEEE/EMBS Conference on Neural Engineering (San Diego, CA: NER), 140-143. doi: 10.1109/NER.2013.6695891

Brem, A. K., Fried, P. J., Horvath, J. C., Robertson, E. M., and PascualLeone, A. (2014). Is neuroenhancement by noninvasive brain stimulation a net zero-sum proposition? Neuroimage 85(pt 3), 1058-1068. doi: 10.1016/j.neuroimage.2013.07.038

Bressler, S. L., and Menon, V. (2010). Large-scale brain networks in cognition: emerging methods and principles. Trends Cogn. Sci. 14, 277-290. doi: 10.1016/j.tics.2010.04.004

Buchel, C., Coull, J. T., and Friston, K. J. (1999). The predictive value of changes in effective connectivity for human learning. Science 283, 1538-1541. doi: 10.1126/science.283.5407.1538

Cabral, J., Luckhoo, H., Woolrich, M., Joensson, M., Mohseni, H., Baker, A., et al. (2014). Exploring mechanisms of spontaneous functional connectivity in MEG: how delayed network interactions lead to structured amplitude envelopes of band-pass filtered oscillations. Neuroimage 90, 423-435. doi: 10.1016/j.neuroimage.2013.11.047

Cerruti, C., and Schlaug, G. (2009). Anodal transcranial direct current stimulation of the prefrontal cortex enhances complex verbal associative thought. J. Cogn. Neurosci. 21, 1980-1987. doi: 10.1162/jocn.2008.21143

Chi, R. P., and Snyder, A. W. (2011). Facilitate insight by non-invasive brain stimulation. PLoS ONE 6:e16655. doi: 10.1371/journal.pone.0016655

Chi, R. P., and Snyder, A. W. (2012). Brain stimulation enables the solution of an inherently difficult problem. Neurosci. Lett. 515, 121-124. doi: 10.1016/j.neulet.2012.03.012

Chib, V. S., Yun, K., Takahashi, H., and Shimojo, S. (2013). Noninvasive remote activation of the ventral midbrain by transcranial direct current stimulation of prefrontal cortex. Transl. Psychiatry 3, e268. doi: 10.1038/tp.2013.44

Chouinard, P. A., Van Der Werf, Y. D., Leonard, G., and Paus, T. (2003). Modulating neural networks with transcranial magnetic stimulation applied over the dorsal premotor and primary motor cortices. J. Neurophysiol. 90, 1071-1083. doi: 10.1152/jn.01105.2002

Chrysikou, E. G., Hamilton, R. H., Coslett, H. B., Datta, A., Bikson, M., and Thompson-Schill, S. L. (2013). Noninvasive transcranial direct current stimulation over the left prefrontal cortex facilitates cognitive flexibility in tool use. Cogn. Neurosci. 4, 81-89. doi: 10.1080/17588928.2013.768221

Cole, M. W., Laurent, P., and Stocco, A. (2013a). Rapid instructed task learning: a new window into the human brain's unique capacity for flexible cognitive control. Cogn. Affect. Behav. Neurosci. 13, 1-22. doi: 10.3758/s13415-012-0125-7
Cole, M. W., Pathak, S., and Schneider, W. (2010). Identifying the brain's most globally connected regions. Neuroimage 49, 3132-3148. doi: 10.1016/j.neuroimage.2009.11.001

Cole, M. W., Reynolds, J. R., Power, J. D., Repovs, G., Anticevic, A., and Braver, T. S. (2013b). Multi-task connectivity reveals flexible hubs for adaptive task control. Nat. Neurosci. 16, 1348-1355. doi: 10.1038/nn.3470

Cole, M. W., and Schneider, W. (2007). The cognitive control network: integrated cortical regions with dissociable functions. Neuroimage 37, 343-360. doi: 10.1016/j.neuroimage.2007.03.071

Datta, A., Bansal, V., Diaz, J., Patel, J., Reato, D., and Bikson, M. (2009). Gyri-precise head model of transcranial direct current stimulation: improved spatial focality using a ring electrode versus conventional rectangular pad. Brain Stimul. 2, 201-207, 207.el. doi: 10.1016/j.brs.2009.03.005

Davare, M., Lemon, R., and Olivier, E. (2008). Selective modulation of interactions between ventral premotor cortex and primary motor cortex during precision grasping in humans. J. Physiol. 586, 2735-2742. doi: 10.1113/ jphysiol.2008.152603

Deans, J. K., Powell, A. D., and Jefferys, J. G. (2007). Sensitivity of coherent oscillations in rat hippocampus to AC electric fields. J. Physiol. 583, 555-565. doi: 10.1113/jphysiol.2007.137711

Delorme, A., and Makeig, S. (2004). EEGLAB: an open source toolbox for analysis of single-trial EEG dynamics including independent component analysis. J. Neurosci. Methods 134, 9-21. doi: 10.1016/j.jneumeth.2003.10.009

Ewald, A., Marzetti, L., Zappasodi, F., Meinecke, F. C., and Nolte, G. (2012). Estimating true brain connectivity from EEG/MEG data invariant to linear and static transformations in sensor space. Neuroimage 60, 476-488. doi: 10.1016/j.neuroimage.2011.11.084

Faria, P., Hallett, M., and Miranda, P. C. (2011). A finite element analysis of the effect of electrode area and inter-electrode distance on the spatial distribution of the current density in tDCS. J. Neural Eng. 8:066017. doi: 10.1088/1741$2560 / 8 / 6 / 066017$

Fornito, A., Zalesky, A., and Breakspear, M. (2013). Graph analysis of the human connectome: promise, progress, and pitfalls. Neuroimage 80, 426-444. doi: 10.1016/j.neuroimage.2013.04.087

Fregni, F., Boggio, P. S., Nitsche, M., Bermpohl, F., Antal, A., Feredoes, E., et al. (2005). Anodal transcranial direct current stimulation of prefrontal cortex enhances working memory. Exp. Brain Res. 166, 23-30. doi: 10.1007/s00221005-2334-6

Friston, K. J. (2011). Functional and effective connectivity: a review. Brain Connect. 1, 13-36. doi: 10.1089/brain.2011.0008

Frohlich, F., and McCormick, D. A. (2010). Endogenous electric fields may guide neocortical network activity. Neuron 67, 129-143. doi: 10.1016/j.neuron.2010.06.005

Frohlich, F., and Schmidt, S. L. (2013). Rational design of transcranial current stimulation (TCS) through mechanistic insights into cortical network dynamics. Front. Hum. Neurosci. 7:804. doi: 10.3389/fnhum.2013.00804

Fuster, J. M. (2000). The module: crisis of a paradigm. Neuron 26, 51-53. doi: 10.1016/S0896-6273(00)81137-X

Gartside, I. B. (1968). Mechanisms of sustained increases of firing rate of neurons in the rat cerebral cortex after polarization: reverberating circuits or modification of synaptic conductance? Nature 220, 382-383. doi: 10.1038/220382a0

Goni, J., van den Heuvel, M. P., Avena-Koenigsberger, A., Velez De Mendizabal, N., Betzel, R. F., Griffa, A., et al. (2014). Resting-brain functional connectivity predicted by analytic measures of network communication. Proc. Natl. Acad. Sci. U.S.A. 111, 833-838. doi: 10.1073/pnas.1315529111

Goswami, U. (2000). Phonological representations, reading development and dyslexia: towards a cross-linguistic theoretical framework. Dyslexia 6, 133-151. doi: 10.1002/(sici) 1099-0909(200004/06)6:2<133::aid-dys160>3.0.co;2-a

Hasan, A., Bergener, T., Nitsche, M. A., Strube, W., Bunse, T., Falkai, P., et al. (2013). Impairments of motor-cortex responses to unilateral and bilateral direct current stimulation in schizophrenia. Front. Psychiatry 4:121. doi: 10.3389/fpsyt.2013.00121

Horwitz, B. (2003). The elusive concept of brain connectivity. Neuroimage 19, 466-470. doi: 10.1016/S1053-8119(03)00112-5

Jaušovec, N., and Jaušovec, K. (2000a). Differences in resting EEG related to ability. Brain Topogr. 12, 229-240. doi: 10.1023/A:1023446024923

Jaušovec, N., and Jaušovec, K. (2000b). EEG activity during the performance of complex mental problems. Int. J. Psychophysiol. 36, 73-88. doi: 10.1016/S01678760(99)00113-0 
Jung, R. E., Grazioplene, R., Caprihan, A., Chavez, R. S., and Haier, R. J. (2010a). White matter integrity, creativity, and psychopathology: disentangling constructs with diffusion tensor imaging. PLoS ONE 5:e9818. doi: 10.1371/journal.pone.0009818

Jung, R. E., Segall, J. M., Jeremy Bockholt, H., Flores, R. A., Smith, S. M., Chavez, R. S., et al. (2010b). Neuroanatomy of creativity. Hum. Brain Mapp. 31, 398-409. doi: $10.1002 / \mathrm{hbm} .20874$

Jung-Beeman, M., Bowden, E. M., Haberman, J., Frymiare, J. L., Arambel-Liu, S., Greenblatt, R., et al. (2004). Neural activity when people solve verbal problems with insight. PLoS Biol. 2:E97. doi: 10.1371/journal.pbio.0020097

Kanai, R., Paulus, W., and Walsh, V. (2010). Transcranial alternating current stimulation (tACS) modulates cortical excitability as assessed by TMSinduced phosphene thresholds. Clin. Neurophysiol. 121, 1551-1554. doi: 10.1016/j.clinph.2010.03.022

Kanwisher, N., McDermott, J., and Chun, M. M. (1997). The fusiform face area: a module in human extrastriate cortex specialized for face perception. J. Neurosci. $17,4302-4311$

Kar, K., and Krekelberg, B. (2014). Transcranial alternating current stimulation attenuates visual motion adaptation. J. Neurosci. 34, 7334-7340. doi 10.1523/JNEUROSCI.5248-13.2014

Kar, K., and Wright, J. (2014). Probing the mechanisms underlying the mitigation of cognitive aging with anodal transcranial direct current stimulation. J. Neurophysiol. 111, 1397-1399. doi: 10.1152/jn.00736.2013

Keeser, D., Meindl, T., Bor, J., Palm, U., Pogarell, O., Mulert, C., et al. (2011). Prefrontal transcranial direct current stimulation changes connectivity of resting-state networks during fMRI. J. Neurosci. 31, 15284-15293. doi: 10.1523/JNEUROSCI.0542-11.2011

Kounios, J., Fleck, J., Green, D., Payne, L., Stevenson, J. L., Bowden, E. M., et al. (2008). The origins of insight in resting-state brain activity. Neuropsychologia 46, 281-291. doi: 10.1016/j.neuropsychologia.2007.07.013

Kwon, Y. H., Ko, M. H., Ahn, S. H., Kim, Y. H., Song, J. C., Lee, C. H., et al. (2008). Primary motor cortex activation by transcranial direct current stimulation in the human brain. Neurosci. Lett. 435, 56-59. doi: 10.1016/j.neulet.2008.02.012

Lang, N., Siebner, H. R., Ward, N. S., Lee, L., Nitsche, M. A., Paulus, W., et al. (2005). How does transcranial DC stimulation of the primary motor cortex alter regional neuronal activity in the human brain? Eur. J. Neurosci. 22, 495-504. doi: 10.1111/j.1460-9568.2005.04233.x

Lehnertz, K., Ansmann, G., Bialonski, S., Dickten, H., Geier, C., and Porz, S. (2014) Evolving networks in the human epileptic brain. Physica D Nonlin. Phenom. 267, 7-15. doi: 10.1016/j.physd.2013.06.009

Liebetanz, D., Nitsche, M. A., Tergau, F., and Paulus, W. (2002). Pharmacological approach to the mechanisms of transcranial DC-stimulation-induced aftereffects of human motor cortex excitability. Brain 125, 2238-2247. doi: 10.1093/brain/awf238

Luft, C. D. B., and Bhattacharya, J. (2014). "Correlatos neurales de la creatividad," in Conectividad Funcional y Anatómica en el Crebro Humano: Análisis De Señales y Aplicaciones en Ciencias de la Salud, eds F. Maestú, E. Pereda, and F. Del Pozo (Madrid: Elsevier), (in press).

Malenka, R. C., and Bear, M. F. (2004). LTP and LTD: an embarrassment of riches. Neuron 44, 5-21. doi: 10.1016/j.neuron.2004.09.012

Massimini, M., Ferrarelli, F., Huber, R., Esser, S. K., Singh, H., and Tononi, G. (2005). Breakdown of cortical effective connectivity during sleep. Science 309, 2228-2232. doi: 10.1126/science.1117256

Meinzer, M., Antonenko, D., Lindenberg, R., Hetzer, S., Ulm, L., Avirame, K., et al. (2012). Electrical brain stimulation improves cognitive performance by modulating functional connectivity and task-specific activation. J. Neurosci. 32, 1859-1866. doi: 10.1523/JNEUROSCI.4812-11.2012

Meinzer, M., Lindenberg, R., Antonenko, D., Flaisch, T., and Floel, A. (2013). Anodal transcranial direct current stimulation temporarily reverses ageassociated cognitive decline and functional brain activity changes. J. Neurosci. 33, 12470-12478. doi: 10.1523/JNEUROSCI.5743-12.2013

Metuki, N., Sela, T., and Lavidor, M. (2012). Enhancing cognitive control components of insight problems solving by anodal tDCS of the left dorsolateral prefrontal cortex. Brain Stimul. 5, 110-115. doi: 10.1016/j.brs.2012.03.002

Mochizuki, H., Huang, Y. Z., and Rothwell, J. C. (2004). Interhemispheric interaction between human dorsal premotor and contralateral primary motor cortex. J. Physiol. 561, 331-338. doi: 10.1113/jphysiol.2004.072843

Moliadze, V., Antal, A., and Paulus, W. (2010). Electrode-distance dependent after-effects of transcranial direct and random noise stimulation with extracephalic reference electrodes. Clin. Neurophysiol. 121, 2165-2171. doi: 10.1016/j.clinph.2010.04.033

Monte-Silva, K., Kuo, M. F., Hessenthaler, S., Fresnoza, S., Liebetanz, D., Paulus, W., et al. (2013). Induction of late LTP-like plasticity in the human motor cortex by repeated non-invasive brain stimulation. Brain Stimul. 6, 424-432. doi: 10.1016/j.brs.2012.04.011

Mordillo-Mateos, L., Turpin-Fenoll, L., Millan-Pascual, J., Nunez-Perez, N., Panyavin, I., Gomez-Arguelles, J. M., et al. (2012). Effects of simultaneous bilateral tDCS of the human motor cortex. Brain Stimul. 5, 214-222. doi: 10.1016/j.brs.2011.05.001

Morishima, Y., Akaishi, R., Yamada, Y., Okuda, J., Toma, K., and Sakai, K. (2009). Task-specific signal transmission from prefrontal cortex in visual selective attention. Nat. Neurosci. 12, 85-91. doi: 10.1038/nn.2237

Neuling, T., Rach, S., and Herrmann, C. S. (2013). Orchestrating neuronal networks: sustained after-effects of transcranial alternating current stimulation depend upon brain states. Front. Hum. Neurosci. 7:161. doi: 10.3389/fnhum.2013.00161

Niso, G., Bruna, R., Pereda, E., Gutierrez, R., Bajo, R., Maestu, F., et al. (2013). HERMES: towards an integrated toolbox to characterize functional and effective brain connectivity. Neuroinformatics 11, 405-434. doi: 10.1007/s12021-0139186-1

Nitsche, M. A., Boggio, P. S., Fregni, F., and Pascual-Leone, A. (2009). Treatment of depression with transcranial direct current stimulation (tDCS): a review. Exp. Neurol. 219, 14-19. doi: 10.1016/j.expneurol.2009.03.038

Nitsche, M. A., Cohen, L. G., Wassermann, E. M., Priori, A., Lang, N., Antal, A., et al. (2008). Transcranial direct current stimulation: state of the art 2008. Brain Stimul. 1, 206-223. doi: 10.1016/j.brs.2008.06.004

Nitsche, M. A., Fricke, K., Henschke, U., Schlitterlau, A., Liebetanz, D., Lang, N., et al. (2003). Pharmacological modulation of cortical excitability shifts induced by transcranial direct current stimulation in humans. J. Physiol. 553, 293-301. doi: 10.1113/jphysiol.2003.049916

Nitsche, M. A., and Paulus, W. (2000). Excitability changes induced in the human motor cortex by weak transcranial direct current stimulation. J. Physiol. 527(pt 3), 633-639. doi: 10.1111/j.1469-7793.2000.t01-1-00633.x

Nitsche, M. A., and Paulus, W. (2001). Sustained excitability elevations induced by transcranial DC motor cortex stimulation in humans. Neurology 57, 1899-1901. doi: 10.1212/WNL.57.10.1899

Oostenveld, R., Fries, P., Maris, E., and Schoffelen, J. M. (2011). FieldTrip: open source software for advanced analysis of MEG, EEG, and invasive electrophysiological data. Comput. Intell. Neurosci. 2011:156869. doi: 10.1155/2011/ 156869

O'Shea, J., Johansen-Berg, H., Trief, D., Gobel, S., and Rushworth, M. F. (2007) Functionally specific reorganization in human premotor cortex. Neuron 54, 479-490. doi: 10.1016/j.neuron.2007.04.021

Parra, L. C., and Bikson, M. (2004). Model of the effect of extracellular fields on spike time coherence. Conf. Proc. IEEE Eng. Med. Biol. Soc. 6, 4584-4587. doi: 10.1109/IEMBS.2004.1404271

Pascual-Leone, A., and Walsh, V. (2001). Fast backprojections from the motion to the primary visual area necessary for visual awareness. Science 292, 510-512. doi: 10.1126/science.1057099

Pena-Gomez, C., Sala-Lonch, R., Junque, C., Clemente, I. C., Vidal, D., Bargallo, N., et al. (2012). Modulation of large-scale brain networks by transcranial direct current stimulation evidenced by resting-state functional MRI. Brain Stimul. 5, 252-263. doi: 10.1016/j.brs.2011.08.006

Pereda, E., Quiroga, R. Q., and Bhattacharya, J. (2005). Nonlinear multivariate analysis of neurophysiological signals. Prog. Neurobiol. 77, 1-37. doi: 10.1016/j.pneurobio.2005.10.003

Plucker, J. A., Beghetto, R. A., and Dow, G. T. (2004). Why isn't creativity more important to educational psychologists? Potentials, pitfalls, and future directions in creativity research. Educ. Psychol. 39, 83-96. doi: 10.1207/s15326985ep3902_1

Polania, R., Nitsche, M. A., and Paulus, W. (2011a). Modulating functional connectivity patterns and topological functional organization of the human brain with transcranial direct current stimulation. Hum. Brain Mapp. 32, 1236-1249. doi: $10.1002 / \mathrm{hbm} .21104$

Polania, R., Paulus, W., Antal, A., and Nitsche, M. A. (2011b). Introducing graph theory to track for neuroplastic alterations in the resting human brain: a transcranial direct current stimulation study. Neuroimage 54, 2287-2296. doi: 10.1016/j.neuroimage.2010.09.085 
Polania, R., Paulus, W., and Nitsche, M. A. (2012a). Modulating cortico-striatal and thalamo-cortical functional connectivity with transcranial direct current stimulation. Hum. Brain Mapp. 33, 2499-2508. doi: 10.1002/hbm.21380

Polania, R., Paulus, W., and Nitsche, M. A. (2012b). Reorganizing the intrinsic functional architecture of the human primary motor cortex during rest with non-invasive cortical stimulation. PLoS ONE 7:e30971. doi: 10.1371/journal.pone.0030971

Purpura, D. P., and McMurtry, J. G. (1965). Intracellular activities and evoked potential changes during polarization of motor cortex. J. Neurophysiol. 28, 166-185.

Radman, T., Ramos, R. L., Brumberg, J. C., and Bikson, M. (2009). Role of cortical cell type and morphology in subthreshold and suprathreshold uniform electric field stimulation in vitro. Brain Stimul. 2, 215-228, 228. e1-e3. doi: 10.1016/j.brs.2009.03.007

Rahman, A., Reato, D., Arlotti, M., Gasca, F., Datta, A., Parra, L. C., et al. (2013). Cellular effects of acute direct current stimulation: somatic and synaptic terminal effects. J. Physiol. 591, 2563-2578. doi: 10.1113/jphysiol.2012.247171

Razumnikova, O. M., and Larina, E. N. (2005). Hemispheric interactions during a search of original verbal associations: EEG coherence in creative men and women. Zh. Vyssh. Nerv. Deiat. Im. I P Pavlova 55, 777-787.

Reato, D., Rahman, A., Bikson, M., and Parra, L. C. (2010). Low-intensity electrical stimulation affects network dynamics by modulating population rate and spike timing. J. Neurosci. 30, 15067-15079. doi: 10.1523/JNEUROSCI.2059-10.2010

Rhodes, M. (1961). An analysis of creativity. Phi Delta Kappan 42, 305-310.

Rigonatti, S. P., Boggio, P. S., Myczkowski, M. L., Otta, E., Fiquer, J. T., Ribeiro, R. B., et al. (2008). Transcranial direct stimulation and fluoxetine for the treatment of depression. Eur. Psychiatry 23, 74-76. doi: 10.1016/i.eurpsy.2007.09.006

Rioult-Pedotti, M. S., Friedman, D., and Donoghue, J. P. (2000). Learninginduced LTP in neocortex. Science 290, 533-536. doi: 10.1126/science.290. 5491.533

Rubinov, M., and Sporns, O. (2010). Complex network measures of brain connectivity: uses and interpretations. Neuroimage 52, 1059-1069. doi: 10.1016/j.neuroimage.2009.10.003

Ruffini, G., Wendling, F., Merlet, I., Molaee-Ardekani, B., Mekonnen, A., Salvador, R., et al. (2013). Transcranial current brain stimulation (tCS): models and technologies. IEEE Trans. Neural Syst. Rehabil. Eng. 21, 333-345. doi: 10.1109/TNSRE.2012.2200046

Santiesteban, I., Banissy, M. J., Catmur, C., and Bird, G. (2012). Enhancing social ability by stimulating right temporoparietal junction. Curr. Biol. 22, 2274-2277. doi: 10.1016/j.cub.2012.10.018

Sawyer, K. (2012). Explaining Creativity: The Science of Human Innovation. New York, NY: Oxford University Press, Inc.

Schaal, N. K., Williamson, V. J., and Banissy, M. J. (2013). Anodal transcranial direct current stimulation over the supramarginal gyrus facilitates pitch memory. Eur. J. Neurosci. 38, 3513-3518. doi: 10.1111/ejn.12344

Sehm, B., Kipping, J., Schafer, A., Villringer, A., and Ragert, P. (2013). A comparison between uni- and bilateral tDCS effects on functional connectivity of the human motor cortex. Front. Hum. Neurosci. 7:183. doi: 10.3389/fnhum.2013.00183

Shafi, M. M., Westover, M. B., Fox, M. D., and Pascual-Leone, A. (2012). Exploration and modulation of brain network interactions with noninvasive brain stimulation in combination with neuroimaging. Eur. J. Neurosci. 35, 805-825. doi: 10.1111/j.1460-9568.2012.08035.x

Shahid, S. S., Bikson, M., Salman, H., Wen, P., and Ahfock, T. (2014). The value and cost of complexity in predictive modelling: role of tissue anisotropic conductivity and fibre tracts in neuromodulation. J. Neural Eng. 11:036002. doi: 10.1088/1741-2560/11/3/036002

Snowball, A., Tachtsidis, I., Popescu, T., Thompson, J., Delazer, M., Zamarian, L., et al. (2013). Long-term enhancement of brain function and cognition using cognitive training and brain stimulation. Curr. Biol. 23, 987-992. doi: 10.1016/j.cub.2013.04.045

Sporns, O. (2011). Networks of the Brain. Cambridge, MA: MIT Press.

Sporns, O. (2014). Contributions and challenges for network models in cognitive neuroscience. Nat. Neurosci. 17, 652-660. doi: 10.1038/nn.3690
Sporns, O., Chialvo, D. R., Kaiser, M., and Hilgetag, C. C. (2004). Organization, development and function of complex brain networks. Trends Cogn. Sci. 8, 418-425. doi: 10.1016/j.tics.2004.07.008

Takano, Y., Yokawa, T., Masuda, A., Niimi, J., Tanaka, S., and Hironaka, N. (2011). A rat model for measuring the effectiveness of transcranial direct current stimulation using fMRI. Neurosci. Lett. 491, 40-43. doi: 10.1016/j.neulet.2011.01.004

Takeuchi, H., Taki, Y., Hashizume, H., Sassa, Y., Nagase, T., Nouchi, R., et al. (2012). The association between resting functional connectivity and creativity. Cereb. Cortex 22, 2921-2929. doi: 10.1093/cercor/bhr371

Takeuchi, H., Taki, Y., Sassa, Y., Hashizume, H., Sekiguchi, A., Fukushima, A., et al. (2010). White matter structures associated with creativity: evidence from diffusion tensor imaging. Neuroimage 51, 11-18. doi: 10.1016/j.neuroimage.2010. 02.035

Terao, Y., and Ugawa, Y. (2002). Basic mechanisms of TMS. J. Clin. Neurophysiol. 19, 322-343. doi: 10.1097/00004691-200208000-00006

Terhune, D. B., and Cohen Kadosh, R. (2013). Modulating cognitive control in major depression with transcranial electrical stimulation. Biol. Psychiatry 73, 595-596. doi: 10.1016/j.biopsych.2013.01.006

Terney, D., Chaieb, L., Moliadze, V., Antal, A., and Paulus, W. (2008). Increasing human brain excitability by transcranial high-frequency random noise stimulation. J. Neurosci. 28, 14147-14155. doi: 10.1523/JNEUROSCI.4248-08.2008

Uehara, T., Yamasaki, T., Okamoto, T., Koike, T., Kan, S., Miyauchi, S., et al. (2014). Efficiency of a "small-world" brain network depends on consciousness level: a resting-state FMRI study. Cereb. Cortex 24, 1529-1539. doi: 10.1093/cercor/bht004

van den Heuvel, M. P., and Sporns, O. (2011). Rich-club organization of the human connectome. J. Neurosci. 31, 15775-15786. doi: 10.1523/JNEUROSCI.353911.2011

van den Heuvel, M. P., and Sporns, O. (2013). Network hubs in the human brain. Trends Cogn. Sci. 17, 683-696. doi: 10.1016/j.tics.2013.09.012

van den Heuvel, M. P., Sporns, O., Collin, G., Scheewe, T., Mandl, R. C., Cahn, W., et al. (2013). Abnormal rich club organization and functional brain dynamics in schizophrenia. JAMA Psychiatry 70, 783-792. doi: 10.1001/jamapsychiatry.2013.1328

Wagner, T., Fregni, F., Fecteau, S., Grodzinsky, A., Zahn, M., and Pascual-Leone, A. (2007). Transcranial direct current stimulation: a computer-based human model study. Neuroimage 35, 1113-1124. doi: 10.1016/j.neuroimage.2007.01.027

Watts, D. J., and Strogatz, S. H. (1998). Collective dynamics of 'small-world' networks. Nature 393, 440-442. doi: 10.1038/30918

Weber, M. J., Messing, S. B., Rao, H., Detre, J. A., and Thompson-Schill, S. L. (2014). Prefrontal transcranial direct current stimulation alters activation and connectivity in cortical and subcortical reward systems: a tDCS-fMRI study. Hum. Brain Mapp. 35, 3673-3686. doi: 10.1002/hbm.22429

Wiggins, G., and Bhattacharya, J. (2014). Mind the gap: an attempt to bridge computational and neuroscientific approaches to study creativity. Front. Hum. Neurosci. 8:540. doi: 10.3389/fnhum.2014.00540

Conflict of Interest Statement: The authors declare that the research was conducted in the absence of any commercial or financial relationships that could be construed as a potential conflict of interest.

Received: 01 June 2014; accepted: 07 July 2014; published online: 30 July 2014. Citation: Luft CDB, Pereda E, Banissy MJ and Bhattacharya J (2014) Best of both worlds: promise of combining brain stimulation and brain connectome. Front. Syst. Neurosci. 8:132. doi: 10.3389/fnsys.2014.00132

This article was submitted to the journal Frontiers in Systems Neuroscience. Copyright (c) 2014 Luft, Pereda, Banissy and Bhattacharya. This is an open-access article distributed under the terms of the Creative Commons Attribution License (CC BY). The use, distribution or reproduction in other forums is permitted, provided the original author(s) or licensor are credited and that the original publication in this journal is cited, in accordance with accepted academic practice. No use, distribution or reproduction is permitted which does not comply with these terms. 